\title{
Broadband absorption enhancement of organic solar cells with interstitial lattice patterned metal nanoparticles
}

\author{
Luzhou Chen, Wallace C. H. Choy, a) and Wei E. I. Sha \\ Department of Electrical and Electronic Engineering, The University of Hong Kong, Pokfulam Road, \\ Hong Kong, China
}

(Received 4 February 2013; accepted 13 June 2013; published online 26 June 2013)

\begin{abstract}
Light blocking induced by top patterned nanostructures is a fundamental limit in solar cells absorption. Here we propose an interstitial lattice patterned organic solar cell which can improve the light blocking of traditional square lattice and achieve broadband absorption enhancement. Compared to square lattice design, the plasmonic mode couplings between individual metallic nanoparticles in the interstitial lattice are more versatile and much stronger. Moreover, plasmonic modes can couple to the guided modes, resulting in large enhancement factor at some wavelengths. The interstitial lattice concept will be a broad interest and great help for high-performance photovoltaics. (C) 2013 AIP Publishing LLC. [http://dx.doi.org/10.1063/1.4812517]
\end{abstract}

Organic solar cells (OSCs) have attracted intense attention due to their advantages of low cost, easy fabrication, and high flexibility compared to its inorganic counterparts. However, due to the conflicts between the short diffusion length of excitons and long absorption length of incident photons, the thickness of OSCs is typically thin, and thus power conversion efficiency (PCE) is generally lower than traditional silicon solar cells. Therefore, an exquisite design of light trapping schemes is essential to the PCE improvement of OSCs. Recently, plasmonic effects of metal nanostructures which can enhance the light absorption have been extensively explored. ${ }^{1-20}$ Metal grating is one of widely studied structures where the vertically incident energy can be redirected horizontally and trapped inside the active layer due to excitation of surface plasmon polariton (SPP) mode, leading to strong absorption enhancement. ${ }^{1-10}$ Another popular plasmonic structure is the metal nanoparticles (NPs) which can excite localized surface plasmonic (LSP) mode with near field concentration. ${ }^{11-20}$

However, for both metal NPs and gratings, there is a concern of the fundamental limit of light blocking (i.e., reflection and absorption) when deposit the metal nanostructures on the top of the active layer from which the light will propagate into the solar cells. As a result, the energy penetrating into the solar cell architecture will be dramatically reduced. Motivated by this issue, we propose the concept of interstitial lattice where two sets of square lattices interpenetrated with each other (Fig. 1). Critically different from the widely studied simple lattices such as square or triangular lattice, the interstitial lattice is a kind of compound lattice comprising two particles in a unit cell. ${ }^{21}$ Our numerical results confirm that interstitial lattice patterned OSCs can achieve broadband absorption enhancement compared with the commonly applied square lattice design. The reduced light blocking by the interstitial lattice benefits from much stronger and more versatile plasmonic couplings between the metal NPs. The well balance between light absorption enhancement and light blocking is a unique property of the

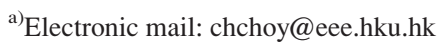

interstitial lattice. Besides, the versatile and strong plasmonic modes coupled to guided mode in the active layer offer large absorption enhancement in some wavelengths region.

Our proposed OSC structure (Fig. 1(a)) comprising interstitial lattice patterned $\mathrm{Ag}$ nano-cylinders embedded in the poly(3,4-ethylenedioxythiophene):poly(styrenesulfonate) (PEDOT:PSS) buffer layer on top of the poly(3-hexylthiophene):[6,6]-phenyl C61-butyric acid methyl ester (P3HT: PCBM) active layer. The interstitial lattice pattern is formed by two interpenetrating square lattices as illustrated in Fig. 1(b). As a comparison, commonly applied square lattice pattern is shown in Fig. 1(c). This design is experimentally feasible by the electron beam lithography (EBL) and lift-off technique. Due to its high deflection capabilities, EBL can access large scale arrays with very small NP sizes. ${ }^{22}$ In simulation, we employ complex refractive index of P3HT:PCBM and PEDOT:PSS determined from ellipsometer measurement. $^{23}$ The complex refractive index of $\mathrm{Ag}$ and $\mathrm{Al}$ is calculated by Brendel-Bormann model. ${ }^{24}$ The structure is simulated based on vector Finite-Element method with edge

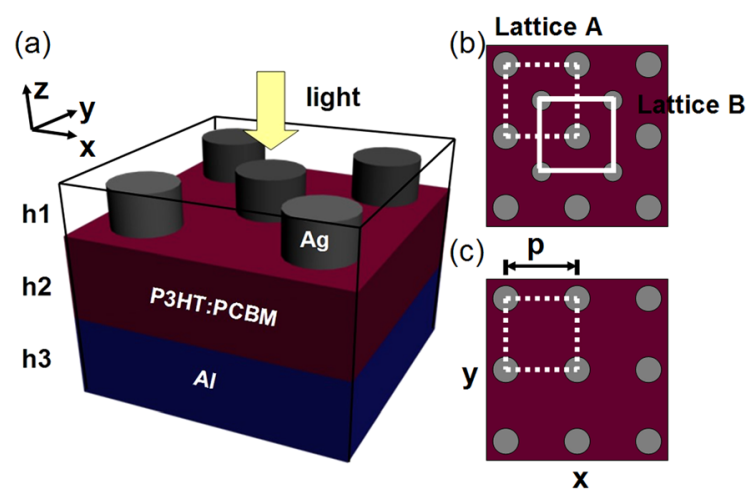

FIG. 1. (a) Schematic illustration of OSC with interstitial lattice patterned Ag nano-cylinders embedded in PEDOT:PSS. Thickness of Ag/PEDOT:PSS layer h1 = $10 \mathrm{~nm}$, P3HT:PCBM absorption layer h2 $=100 \mathrm{~nm}$, and Al layer $\mathrm{h} 3=100 \mathrm{~nm}$. Normal incident light points to the negative $\mathrm{z}$ direction, polarized along the y direction. (b) Interstitial lattice pattern formed by two sets of square lattices: lattice $\mathrm{A}$ is denoted as dot line and lattice $\mathrm{B}$ is denoted as solid line. (c) Square lattice pattern. For both interstitial lattice and square lattice, the length of a unit cell is $\mathrm{p}$. 
elements. In order to calculate an infinitely extended structure, periodic boundary conditions based on the Floquet theorem are applied to the unit cell. At the top and bottom regions we use second-order absorption boundary condition to simulate the semi-infinite air regions. To guarantee the accuracy of the numerical calculation, scattering matrix method $^{25-27}$ is employed for benchmark calculation. Meanwhile, in order to remove the lossy absorption which does not contribute to the power generation of solar cells especially the metal structures such as the Ag NPs and Al substrate, we extract the absorption power $A(\lambda)$ of the active layer in device structure by taking volume integral

$$
A(\lambda)=\int_{V_{0}} \frac{2 \pi c}{\lambda} \varepsilon_{0} n(\lambda) k(\lambda)|\mathbf{E}(r, \lambda)|^{2} d V,
$$

where $\mathbf{E}$ is the electric field at the active layer, $V_{o}$ denotes the total volume of polymer materials of the active layer, $n$ and $k$ refer to the real and imaginary parts of complex refractive index of P3HT:PCBM, $\lambda$ is the wavelength of incident light, and $\varepsilon_{0}$ is the vacuum dielectric constant. Absorptivity of the OSC is defined as $A(\lambda) / A_{0}$, where $A_{0}$ is the total incident power.

In modeling, thickness of the Ag/PEDOT:PSS buffer layer is $\mathrm{h} 1=10 \mathrm{~nm}$, P3HT:PCBM h2 $=100 \mathrm{~nm}$, and Al substrate $\mathrm{h} 3=100 \mathrm{~nm}$. The two interpenetrated square lattices of the interstitial lattice pattern are denoted as lattice A and lattice B (Fig. 1(b)) with corresponding radius of the nanocylinders as $R_{A}$ and $R_{B}$. Due to the symmetry of the studied patterns for $\mathrm{p}$ - and s-polarized normally incident light, we simply only consider polarization direction along the y-axis as shown in Fig. 1(a). Absorptivity of interstitial lattice patterned OSC $\left(R_{A}=R_{B}=10 \mathrm{~nm}, p=70 \mathrm{~nm}\right)$, square lattice patterned $\mathrm{OSC}(\mathrm{R}=10 \mathrm{~nm}, \mathrm{p}=70 \mathrm{~nm})$, and unpatterned planar OSC are shown in Fig. 2(a), and corresponding
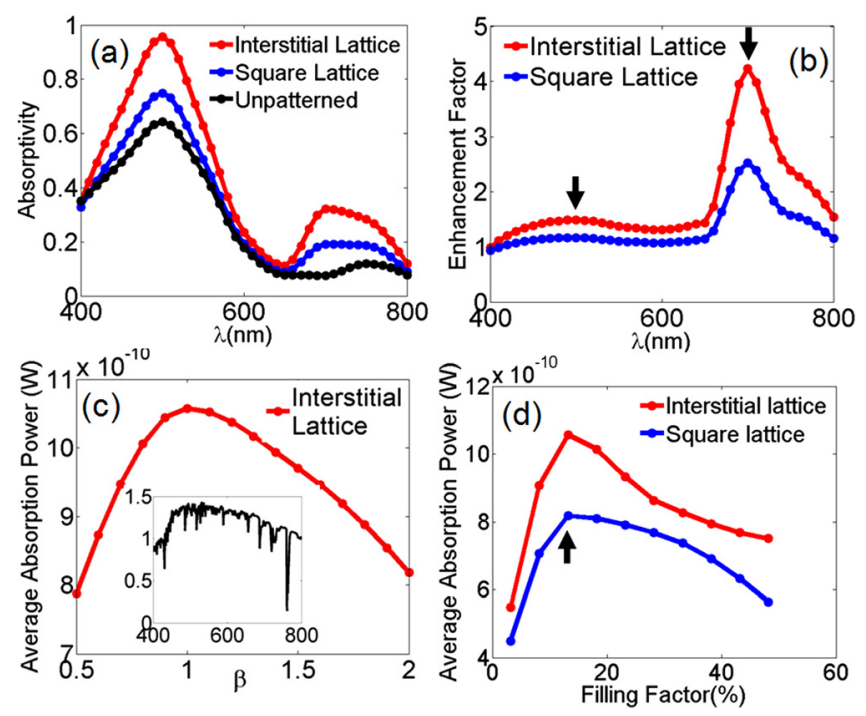

FIG. 2. (a) Absorptivity of interstitial lattice patterned OSCs with $\mathrm{R}_{\mathrm{A}}=\mathrm{R}_{\mathrm{B}}=10 \mathrm{~nm}, \mathrm{p}=70 \mathrm{~nm}$, square lattice with $\mathrm{R}=10 \mathrm{~nm}, \mathrm{p}=70 \mathrm{~nm}$, and planar unpatterned OSC. (b) Enhancement factor of the absorptivity of patterned OSCs. Two enhancement peaks are pointed out by arrows. (c) Average absorption power of interstitial lattice vs. $\beta$. Inset figure is the standard sun irradiation spectrum (AM 1.5). (d) Average absorption power of interstitial lattice vs. $f f$ and result of square lattice. Maximum limit value of average absorption power of square lattice is located by the arrow. enhancement factor is shown in Fig. 2(b). Here enhancement factor is defined as the ratio of the absorptivity of patterned OSC over that of planar unpatterned OSCs. It can be observed that the interstitial lattice OSC can achieve high and broadband enhancement in the whole investigated wavelength region of $400-800 \mathrm{~nm}$. Meanwhile, there are two obvious enhancement peaks at around $500 \mathrm{~nm}$ and $700 \mathrm{~nm}$, reaching enhancement factor of 1.49 and 4.24 , respectively. These enhancements are important and useful because $500 \mathrm{~nm}$ is the central wavelength of the absorption coefficient of P3HT:PCBM. The long wavelength peak of $700 \mathrm{~nm}$ is the wavelength near to the absorption band edge where the incident photon requires longer absorption length, and thus the device typically suffers from weak absorption due to the thin active layer. With our interstitial lattice structure, we cannot only counter-compensate the intrinsic weak absorption but also offer enhancement with peak at $700 \mathrm{~nm}$.

The optical properties of period structures strongly depend on size and period of the metal nanostructures. In our study, the period of the Ag pattern is chosen to be $p=70 \mathrm{~nm}$ because longer distance will weaken the electromagnetic coupling between the NPs and thus reduce the overall plasmonic enhancement effect. However, for the case with closer pattern, the incident light will be shaded and also result in lower performance. To investigate the effect of the NP size, weighted average absorption power of OSCs is calculated by

$$
\langle A\rangle=\frac{\int_{400 \mathrm{~nm}}^{800 \mathrm{~nm}} A(\lambda) \frac{d I(\lambda)}{d \lambda} d \lambda}{\int_{400 \mathrm{~nm}}^{800 \mathrm{~nm}} I(\lambda) d \lambda},
$$

where $A(\lambda)$ is the absorption power of the active layer calculated by Eq. (1) and $I(\lambda)$ is the sun irradiation spectrum intensity of standard air mass 1.5 (inset figure in Fig. 2(c)). The size ratio of the two sublattices is denoted as $\beta=R_{A} / R_{B}$. Average absorption power of OSCs with different $\beta$ in Fig. 2(c) indicates that best performance can be achieved when NPs size of lattice A equals lattice B $(\beta=1)$. Filling factor $(f f)$ of the two kinds of the lattices is also investigated (Fig. 2(d)). For interstitial lattice, $f f=\pi\left(\mathrm{R}_{\mathrm{A}}{ }^{2}+\mathrm{R}_{\mathrm{B}}{ }^{2}\right)$ and for square lattice $f f=\pi R^{2}$. In square lattice patterned OSCs, when the size of NPs is much smaller than the spacing, increasing $f f$ will lead to the increment of average absorption power because larger NPs can enhance LSP effect. After reaching a maximum value, further increasing the NP size will result in decrease of the average absorption power. This limit cannot be exceeded because of light blocking. Interestingly, by introducing interstitial lattice, the average absorption power of OSCs with the same $f f$ is much higher than the case with square lattice, beyond the light blocking limit (arrow in Fig. 2(d)). Finally we choose $R_{A}=R_{B}$ $=10 \mathrm{~nm}$ for the interstitial design.

Next, we explore the underlying absorption enhancement mechanism in our design. Generally, improvement for both interstitial lattice and square lattice compared with unpatterned OSC is due to near field concentration effect by excitation of localized surface plasmonic mode. Since the interstitial lattice contains two Ag NPs in a unit cell while square lattice has only one, the improvement of optical 
absorption by the interstitial lattice should double the square lattice. However, we find that at the wavelength region around the enhancement peak of $\lambda=500 \mathrm{~nm}$, the enhancement factor of the interstitial lattice reaches $49 \%$, which is significantly more than double that of square lattice (i.e., $16 \%$ at $\lambda=500 \mathrm{~nm}$ ) in Fig. 2(b). This indicates that at this wavelength region, there is another special enhancement mechanism besides the LSP effect of individual NP.

In order to investigate the physics, we calculate the near field profile of the $\mathrm{Ag}$ patterned layer as shown in Fig. 3(a) and find that at $500 \mathrm{~nm}$ and $700 \mathrm{~nm}$, the LSP modes excited by different NPs in the interstitial lattice pattern show versatile coupling with each others. As a comparison, coupling effect between the NPs in the square lattice only occurs along the polarization direction of incident light. Regarding the interstitial lattice, two sets of the sub lattice can lead to more versatile coupling not only in polarization direction but also in diagonal one. Remarkably, at these wavelengths region LSP-LSP mode coupling along the diagonal direction can be observed in Fig. 3(a), which greatly enhances the absorption power compared with square lattice pattern. Meanwhile, even when the $f f$ of a square lattice patterned OSC increases to the same as that of the interstitial lattice (Fig. 2(d)), the resulting absorption power of the square lattice patterned OSC still cannot be higher than interstitial lattice case. For some incident wavelengths such as $\lambda=400 \mathrm{~nm}$, the LSP mode cannot be excited significantly and therefore the LSP-LSP coupling cannot be observed in the interstitial lattice. As a result, around $\lambda=400 \mathrm{~nm}$, both interstitial lattice and square lattice designs do not show obvious improvements to unpatterned OSC. Noteworthy, if the NPs are patterned closer in a square lattice, the LSP-LSP coupling will also become stronger. However, the resulting absorption will not be greatly improved owing to light

(a)

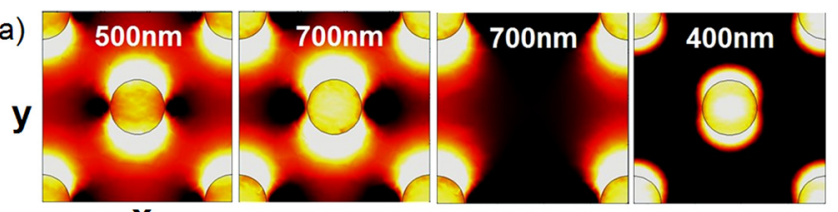

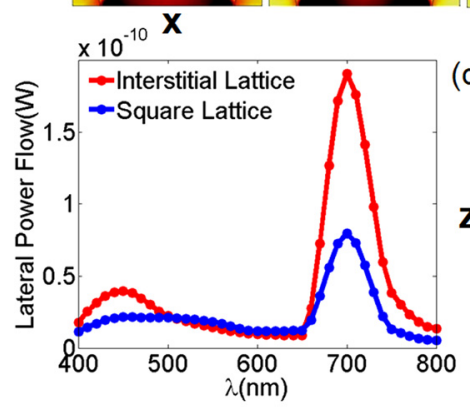

(b)

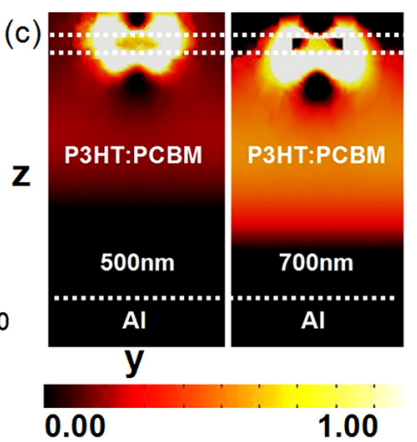

0.00 blocking. With the aid of this interesting property, although more incident light may be blocked for interstitial lattice patterned OSC, it still performs much better than square lattice structure.

It is worth pointing out that the $500 \mathrm{~nm}$ enhancement peaks can be tuned by the NPs size according to the absorption coefficient of active layer material. Here we have chosen $\mathrm{R}_{\mathrm{A}}=\mathrm{R}_{\mathrm{B}}=10 \mathrm{~nm}$ through optimization to set the enhancement peak at $500 \mathrm{~nm}$ for P3HT:PCBM. However, for the other commonly studied absorption materials such as copper phthalocyanine:fullerene $\left(\mathrm{CuPC}: \mathrm{C}_{60}\right)$ with absorption coefficient centered at $600-670 \mathrm{~nm}$, the NP size should be chosen slightly larger because the LSP enhancement peak will redshift as the NP size increase. Consequently, the interstitial pattern design with various NPs size can also be applied for the other solar cells with different absorption material.

Further, we calculate the lateral power flow of the active layer as shown in Fig. 3(b). The sharp peak at $700 \mathrm{~nm}$ of the OSC with interstitial lattice pattern indicates excitation of guided mode at this wavelength. Hence the incident energy can be trapped laterally inside the active layer, leading to strong absorption enhancement. Interestingly, at $700 \mathrm{~nm}$, coupling between the LSP mode excited by the NPs and the guided mode excited in the active layer can be observed from the near field profile in Fig. 3(c), resulting in a hybrid mode which can strongly enhance the long wavelength photon absorption. Differently, no hybrid mode is excited for $\lambda=500 \mathrm{~nm}$. Besides the absorption enhancement due to the LSP-LSP coupling at the top Ag pattern layer, LSP-guided hybrid mode also considerably contributes to the $700 \mathrm{~nm}$ peak, leading to a large enhancement peak of 4.24 at $700 \mathrm{~nm}$, which is much higher than the enhancement at $500 \mathrm{~nm}$. Consequently, through two different types of mode-coupling mechanisms, high absorption enhancement can be achieved in OSCs with interstitial lattice pattern. The above results indicate that the interstitial lattice design is a better choice for top NPs patterned solar cells applications because the comprehensive enhancement effect is much stronger than the degradation effect of light blocking.

Since the field concentration effect of LSP mode strongly depends on the NPs shape, here we also explore the influence of NP geometry in our OSC design. Compared with some other widely applied NP shapes such as diamond column and square column, we find that cylinder NP shows best performance at the main absorption wavelength range of P3HT:PCBM $(400 \mathrm{~nm}-600 \mathrm{~nm})$ as displayed in Fig. 4(a). The inferior performance of diamond and square shape NPs at $400 \mathrm{~nm}-600 \mathrm{~nm}$ is because the LSP induced field concentration mainly localizes near the corners (Fig. 4(b)). However, for circular shape NPs, the enhanced E field surrounding the NPs spreads in a significantly larger area. As a consequence, cylinder NPs perform better than the other two shapes with the same size. This phenomenon can be explained that the curved surface of cylinder is more effective for restoring force on driven electrons and leading to resonance, so higher field amplification of the surrounding region can be achieved. ${ }^{28}$ Apart from the size, spacing and shape of NPs, the local dielectric environment will also affect the LSP mode. Here we calculate the absorption power of an OSC with same patterned structure except for PEDOT:PSS

FIG. 3. (a) Top view of normalized near field profile of interstitial lattice with incident wavelength $500 \mathrm{~nm}, 700 \mathrm{~nm}, 400 \mathrm{~nm}$ and result of square lattice design with incident wavelength $700 \mathrm{~nm}$. (b) Lateral power flow inside the active layer of OSC with interstitial lattice design $\left(R_{A}=10 \mathrm{~nm}\right.$, $\mathrm{R}_{\mathrm{B}}=10 \mathrm{~nm}$ ) and square lattice design $(\mathrm{R}=10 \mathrm{~nm})$. (c) Cross section view of normalized near field profile of interstitial lattice with incident wavelength $500 \mathrm{~nm}$ and $700 \mathrm{~nm}$. All near field profiles adopt the same normalized color bar. Normal incident light points to the negative $\mathrm{z}$ direction, polarized along the y direction. 

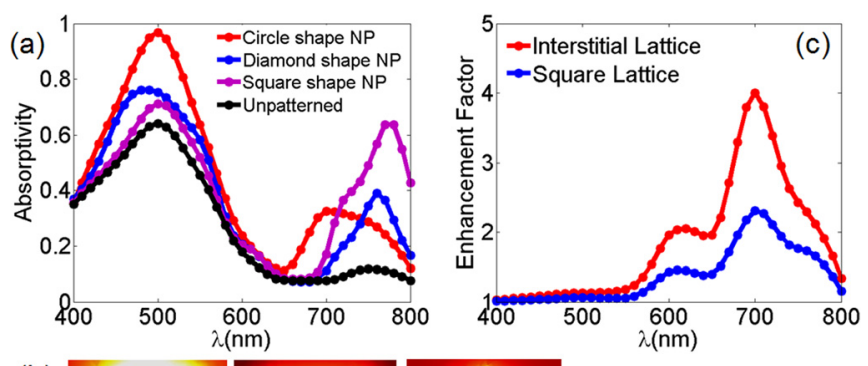

(b)
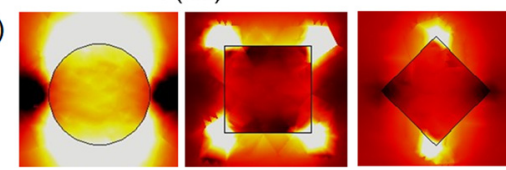

0.00

1.00

FIG. 4. (a) Absorptivity of interstitial lattice patterned OSC with NPs of different shape: circular shapes NPs, diamond shape NPs, square shape NPs, and unpatterned planar OSC. For three different patterns the size of the $\mathrm{Ag}$ NPs are equal to each other. (b) Normalized near field profile of different $\mathrm{Ag}$ NP shapes with incident wavelength $\lambda=500 \mathrm{~nm}$. All near field profiles adopt the same normalized color bar. Normal incident light points to the negative $\mathrm{z}$ direction, polarized along the y direction. (c) Enhancement of absorptivity of interstitial lattice OSC and square lattice OSC using the $\mathrm{Ag} / \mathrm{MoO} 3$ buffer layer. All the geometric parameters are the same as Figs. 2(a) and 2(b).

replaced by Molybdenum trioxide $\left(\mathrm{MoO}_{3}\right)$ with higher refractive index. As shown in Fig. 4(c), the LSP enhanced peak is red-shifted to $610 \mathrm{~nm}$ while that at longer wavelength due to the guided mode is almost unchanged. Therefore, modifications of the dielectric environment of NPs can also be an efficient method to tune the position of the LSP enhancement peak.

In this paper, a top NPs patterned OSC based on the interstitial lattice is proposed. Through the systematical study, we find that by applying this unique design, the LSP modes excited by the NPs can be coupled with each others, leading to strong absorption enhancement. This design can achieve high and broadband absorption improvement compared with the widely applied square lattice pattern. We also find that at some wavelength range, hybrid mode is formed by coupling of LSP mode at the NPs layers and guided mode at the active layer, which will lead to strong enhancement of more than 4 times. NPs of different geometry are also investigated and circular shape has been proved to be the best in the main absorption region of P3HT:PCBM. Our interstitial lattice design proposes a promising method for further improvement of the solar cell absorption as compared to the commonly applied square lattice patterned structure. This work also provides better understanding of the enhancement mechanism by mode-coupling in photovoltaic devices.

This work is supported by University Grant Council of the University of Hong Kong (Grant Nos. 10401466 and
201111159062), the General Research Fund (Grant Nos. HKU\#712010E and HKU711612E), the RGC-NSFC (Grant No. N_HKU709/12), and the SRFDP and RGC ERG Joint Research Scheme (M-HKU703/12) from the Research Grants Council (RGC) of Hong Kong Special Administrative Region, China. The authors acknowledge the useful discussion of D. Zhang.

${ }^{1}$ R. A. Pala, J. White, E. Barnard, J. Liu, and M. L. Brongersma, Adv. Mater. 21, 3504 (2009).

${ }^{2}$ W. Wang, S. Wu, K. Reinhardt, Y. Lu, and S. Chen, Nano Lett. 10, 2012 (2010).

${ }^{3}$ C. Min, J. Li, G. Veronis, J. Y. Lee, S. Fan, and P. Peumans, Appl. Phys. Lett. 96, 133302 (2010).

${ }^{4}$ W. E. I. Sha, W. C. H. Choy, Y. Wu, and W. C. Chew, Opt. Express 20, 2572 (2012).

${ }^{5}$ J. You, X. Li, F. Xie, W. E. I. Sha, J. H. W. Kwong, G. Li, W. C. H. Choy, and Y. Yang, Adv. Energy Mater. 2, 1203 (2012).

${ }^{6}$ X. Li, W. C. H. Choy, L. Huo, F. Xie, W. E. I. Sha, B. Ding, X. Guo, Y. Li, J. Hou, J. You, and Y. Yang, Adv. Mater. 24, 3046 (2012).

${ }^{7}$ X. Li, W. E. I. Sha, W. C. H. Choy, D. D. S. Fung, and F. X. Xie, J. Phys. Chem. C 116, 7200 (2012).

${ }^{8}$ W. E. I. Sha, W. C. H. Choy, and W. C. Chew, Appl. Phys. Lett. 101, 223302 (2012).

${ }^{9}$ K. Tvingstedt, N.-K. Persson, O. Inganas, A. Rahachou, and I. V. Zozoulenko, Appl. Phys. Lett. 91, 113514 (2007).

${ }^{10}$ V. E. Ferry, J. N. Munday, and H. A. Atwater, Adv. Mater. 22, 4794 (2010).

${ }^{11}$ W. Ren, G. Zhang, Y. Wu, H. Ding, Q. Shen, K. Zhang, J. Li, N. Pan, and X. Wang, Opt. Express 19, 26536 (2011).

${ }^{12}$ J. Zhu, M. Xue, H. Shen, Z. Wu, S. Kim, J. J. Ho, A. H. Afshar, B. Zeng, and K. L. Wang, Appl. Phys. Lett. 98, 151110 (2011).

${ }^{13}$ X. Li, W. C. H. Choy, H. Lu, W. E. I. Sha, and A. H. P. Ho, Adv. Funct. Mater. 23, 2728 (2013).

${ }^{14}$ F. X. Xie, W. C. H. Choy, C. C. D. Wang, W. E. I. Sha, and D. D. S. Fung, Appl. Phys. Lett. 99, 153304 (2011).

${ }^{15}$ W. E. I. Sha, W. C. H. Choy, Y. G. Liu, and W. C. Chew, Appl. Phys. Lett. 99, 113304 (2011).

${ }^{16}$ A. J. Morfa, K. L. Rowlen, T. H. Reilly III, M. J. Romero, and J. van de Lagemaat, Appl. Phys. Lett. 92, 013504 (2008).

${ }^{17}$ H. Shen, P. Bienstman, and B. Maes, J. Appl. Phys. 106, 073109 (2009).

${ }^{18}$ J. Yang, J. You, C. C. Chen, W. C. Hsu, H. R. Tan, X. W. Zhang, Z. Hong, and Y. Yang, ACS Nano 5, 6210 (2011).

${ }^{19}$ S. S. Kim, S. I. Na, J. Jo, D. Y. Kim, and Y. C. Nah, Appl. Phys. Lett. 93, 073307 (2008).

${ }^{20}$ M. Westphalen, U. Kreibig, J. Rostalski, H. Lüth, and D. Meissner, Sol. Energy Mater. Sol. Cells 61, 97 (2000).

${ }^{21}$ C. M. Anderson and K. P. Giapis, Phys. Rev. Lett. 77, 2949 (1996).

${ }^{22}$ V. A. Guzenko, J. Ziegler, A. Savouchkina, C. Padeste, and C. David, Microelectron. Eng. 88, 1972 (2011).

${ }^{23}$ D. D. S. Fung, L. Qiao, W. C. H. Choy, C. Wang, W. E. I. Sha, F. Xie, and S. He, J. Mater. Chem. 21, 16349 (2011).

${ }^{24}$ A. D. Rakic, A. B. Djurisic, J. M. Elazar, and M. L. Majewski, Appl. Opt. 37, 5271 (1998).

${ }^{25}$ D. M. Whittaker and I. S. Culshaw, Phys. Rev. B 60, 2610 (1999).

${ }^{26}$ L. Chen, W. E. I. Sha, and W. C. H. Choy, Opt. Express 20, 8175 (2012).

${ }^{27}$ N. A. Gippius, S. G. Tikhodeev, and T. Ishihara, Phys. Rev. B 72, 045138 (2005).

${ }^{28}$ S. A. Maier, Plasmonics: Fundamentals and Applications (Springer, New York, 2007). 